\title{
Re-imagining the Familiar: Data visualisation in environmental art
}

\author{
Lydia Smolin \\ University of Applied Sciences Europe \\ Berlin, Germany \\ lydiasmolin@hotmail.com
}

\section{INTRODUCTION}

This paper explores the idea of how re-imagining familiar images can help transform them and by so doing give them new meaning and power. It focuses on a piece of work entitled 'Endemic Flora 2100 ' which is a data-visualization art installation, designed to captivate the individual regarding the impact of climate change (Figure 1). It discusses how, in the area of visual arts, the application of data-visualization and environmental art can increase climate change awareness by delivering, often complex scientific information in an engaging and visually interesting way.

'Endemic Flora 2100' elevates the endemic endangered flora in the Pirin Mountains, Bulgaria to a new enhanced status by presenting specimens in display cases and jars akin to those found in natural history museums. Thus the formerly familiar is re-imagined in both space and time. (https://vimeo.com/191700507).

\section{ENGAGING METHODS OF PRESENTING ENVIRONMENTAL INFORMATION THROUGH VISUAL ARTS}

In order to spread environmental awareness through the subject of art it is important to look at how information is presented. In particular, for the general public it is difficult to visualise a dataset and can often dilute the impact of its results due to lack of understanding. Using certain methods the viewer can be guided through a subject matter with ease and enjoyment.

If we look at the treatment of an object at the Museum für Naturkunde Berlin (Natural History Museum), particularly 'the Wet Collections' we can see how they preserve and display their specimens in order to create the greatest visual impact on the visitors. One million specimens are stored in 276,000 jars. They are very important from a practical point of view, as the preserved specimens serve to improve conservation, scientific discoveries, biodiversity, and evolution research (Museum für Naturkunde Berlin 2010).

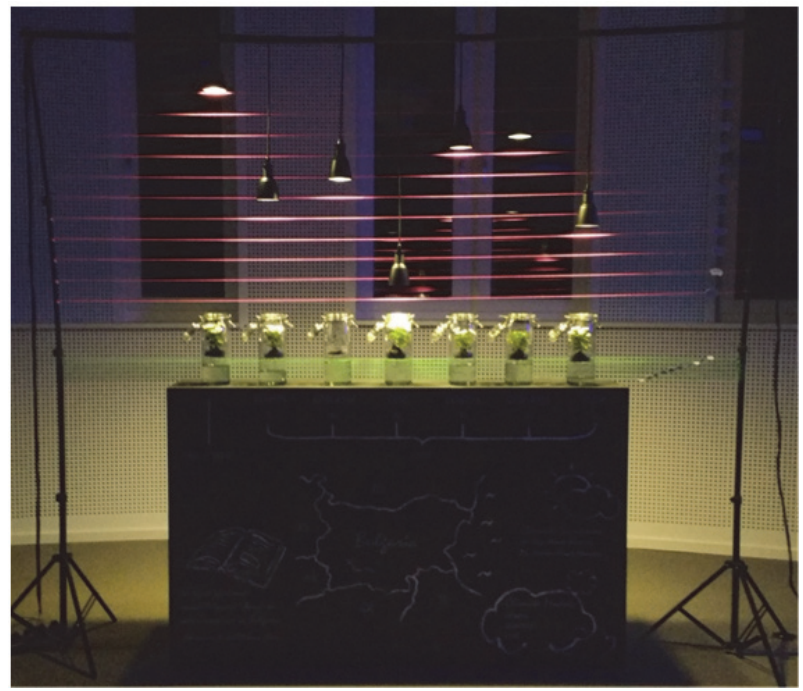

Figure 1: Endemic Flora 2100 installation.

Through a spatial method, Christo and JeanneClaude, the famous environmental art duo always insisted that the purpose of their art is to create works of art for joy and beauty and to create new ways of seeing familiar landscapes. Due to the breath-taking beauty of their artworks and their method of highlighting existing, often, overlooked spaces, they managed to completely change how people viewed and understood those spaces. Both beauty and familiarity are strong factors that can help encourage curiosity (Warde-Aldam 2018).

Using a very tangible approach, Olafur Eliasson and Minik Rosing gathered ice blocks that had detached from Greenland's ice sheet and placed them outside the Tate Modern in London in December 2018, entitled 'Ice Watch'. Time is utilised well here, as the general public are able to witness the physical act of the ice blocks melting. 
They hope through this method that people will become more aware of global warming as we rarely get to see it first-hand (Eliasson \& Rosing 2018).

Re-presenting the familiar by altering an object, in either space or time can re-establish its purpose in a simple, yet effective way, creating new perspectives and insights.

\section{METHOD}

In order to explore how environmental art can effectively highlight climate change, a data visualization installation was created. A dataset was chosen consisting of the two scenarios: A2 high climate warming and B2 - medium climate warming. Only the summer results were chosen, then 3 models in each scenario were selected. This dataset predicts how temperature and precipitation in the Pirin Mountains, Bulgaria might be in the year 2100 .

The installation presents an $\mathrm{H} 110 \times \mathrm{L} 300 \times \mathrm{x} 35 \mathrm{~cm}$ pedestal with six spaces marked out for the six models for the year 2100 and one space for the current reading 1961-1990. A glass jar is placed in each space. A daisy plant representing the chosen endemic flora is suspended inside each glass jar over different levels of water indicating different precipitation levels. A light bulb hangs directly above each jar representing different temperature levels. Pink and green strings are placed behind the jars creating a grid; pink to indicate temperature levels and green to indicate precipitation levels. The light bulbs were placed according to their assigned temperature on the pink grid. The water in each jar was levelled to its assigned height on the green grid. Finally, from the data received each daisy plant was treated differently depending on its model. The treatment of the daisy for the current reading from 1961-1990 remains normal and healthy to stand as a reference to the other models. The other daisy plants each had a different treatment and ranged from diseased, malnourished, not in bloom to extinct. These were carefully analysed taking in all environmental factors and the plant's own unique qualities, thus, predicting plausible outcomes for the year 2100 .

Illustrations were made of each specimen chosen (Figure 2), framed in exhibit cases, lit with soft lighting and labelled with botanic information.

\section{CONCLUSIONS AND DISCUSSION}

In the field of data-visualization, this paper discusses how environmental art can engage with the viewer by re-imagining the familiar, thus, giving new meaning and status to the message. By visualizing the data in a simplistic way using live plants and a visually striking and clear data grid, predicted climate scenarios in the year 2100 are transformed into something tangible and relatable.

The installation succeeded in engaging the public due largely to the simplicity of the grid and the treatment of the jarred plant specimens, which highlighted the predicted outcomes.

The effectiveness of the exhibit could be further enhanced in order to better engage and inform the viewer, by placing illustrations of each specimen on individual pedestals directed at their corresponding scenarios in the jars. Headphones and buttons could be provided. When a button is pushed a light turns on over the specific jar and factual information about the plant, its scenario and its model is explained through headphones.

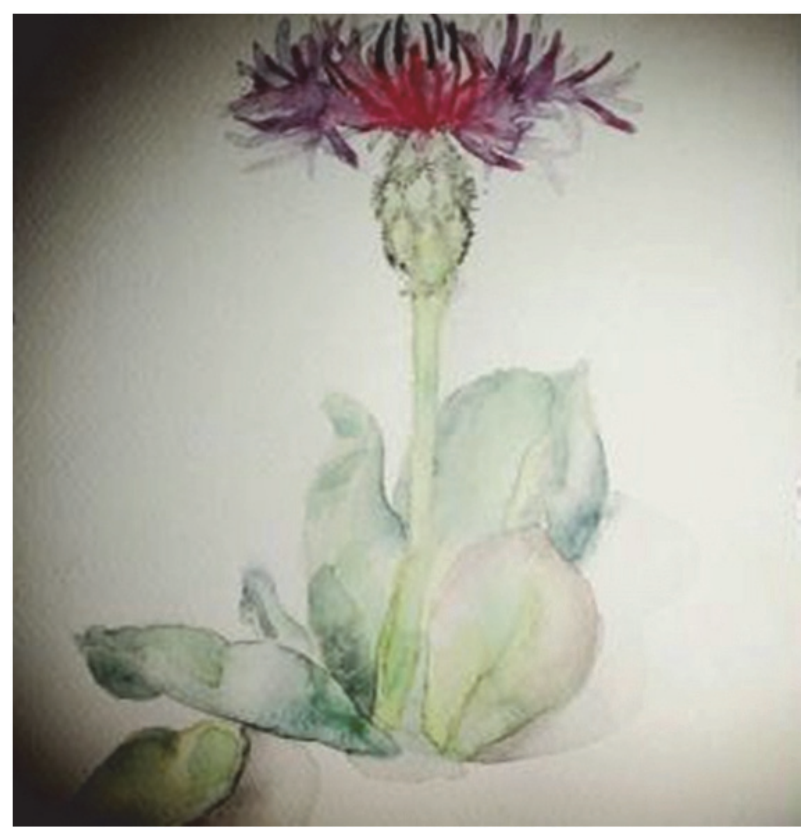

Figure 2: Illustration of endangered specimen.

\section{REFERENCES}

Eliasson, Olafur and Minik Rosing (2018) Ice Watch, 11 Dec 2018, The Tate Modern, London.

Museum für Naturkunde Berlin (2010) The Wet Collections, permanent collection.

Warde-Aldam, D. (2018) Understanding Christo and Jeanne-Claude through 6 Pivotal Artworks. https://www.artsy.net/article/artsy-editorialunderstanding-christo-jeanne-claude-6-pivotalartworks (retrieved 14 Jan 2019). 